\title{
Breast cancer susceptibility: an integrative analysis of genomic data
}

Simone Mocellin, MD, 1-2; Sara Valpione, MD, 3; Carlo Riccardo Rossi, MD, 1-2, Karen Pooley, PhD, 4

1) Istituto Oncologico Veneto (IOV-IRCCS), Padova, Italy

2) Dept. Surgery Oncology and Gastroenterology, University of Padova, Italy

3) CRUK Manchester Institute, The University of Manchester, UK

4) Centre for Cancer Genetic Epidemiology, Dept. Public Health and Primary Care, University of Cambridge, UK

Corresponding author: Simone Mocellin

Istituto Oncologico Veneto (IOV-IRCCS), Padova, Italy

Dept. Surgery Oncology and Gastroenterology, University of Padova, Italy

Via Gattamelata 64

35128 Padova, Italy

E: simone.mocellin@unipd.it

P: 0498215743

F: 0498215575 
bioRxiv preprint doi: https://doi.org/10.1101/279984; this version posted March 12, 2018. The copyright holder for this preprint (which was not certified by peer review) is the author/funder, who has granted bioRxiv a license to display the preprint in perpetuity. It is made available under aCC-BY-NC-ND 4.0 International license.

Key words: breast cancer, susceptibility, risk, predisposition, germline DNA variant, single nucleotide polymorphism, SNP, knowledge-base, integrative analysis, network analysis, pathway analysis, genome-wide association study, GWAS, genomic data.

Abbreviations: GWAS: genome-wide association study; SNP: single nucleotide polymorphism; BCPG: breast cancer predisposition gene; LD: linkage disequilibrium 


\section{Abstract}

\section{Background}

Genome wide association studies (GWAS) are greatly accelerating the pace of discovery of germline variants underlying the genetic architecture of sporadic breast cancer predisposition. We have built the first knowledge-base dedicated to this field and used it to generate hypotheses on the molecular pathways involved in disease susceptibility.

\section{Methods}

We gathered data on the common single nucleotide polymorphisms (SNPs) discovered by breast cancer risk GWAS. Information on SNP functional effect (including data on linkage disequilibrium, expression quantitative trait locus, and SNP relationship with regulatory motifs or promoter/enhancer histone marks) was utilized to select putative breast cancer predisposition genes (BCPGs). Ultimately, BCPGs were subject to pathway (gene set enrichment) analysis and network (protein-protein interaction) analysis.

\section{$\underline{\text { Results }}$}

Data from 38 studies (28 original case-control GWAS enrolling 383,260 patients with breast cancer; and 10 GWAS meta-analyses) were retrieved. Overall, 281 SNPs were associated with the risk of breast cancer with a P-value $<10 \mathrm{E}-06$ and a minor allele frequency $>1 \%$. Based on functional information, we identified 296 putative BCPGs. Primary analysis showed that germline perturbation of classical cancer-related pathways (e.g., apoptosis, cell cycle, signal transduction including estrogen receptor signaling) play a significant role in breast carcinogenesis. Other less established pathways (such as ribosome and peroxisome machineries) were also highlighted. In the main subgroup analysis, we considered the BCPGs encoding transcription factors 
bioRxiv preprint doi: https://doi.org/10.1101/279984; this version posted March 12, 2018. The copyright holder for this preprint (which was not certified by peer review) is the author/funder, who has granted bioRxiv a license to display the preprint in perpetuity. It is made available under aCC-BY-NC-ND 4.0 International license.

$(n=36)$, which in turn target 252 genes. Interestingly, pathway and network analysis of these genes yielded results resembling those of primary analyses, suggesting that most of the effect of genetic variation on disease risk hinges upon transcriptional regulons.

\section{Conclusions}

This knowledge-base, which is freely available and will be annually updated, can inform future studies dedicated to breast cancer molecular epidemiology as well as genetic susceptibility and development. 


\section{Introduction}

With a $10-12 \%$ lifetime risk, breast cancer is the most common cancer among women with about $1,700,000$ new cases and more than 500,000 deaths each year worldwide (1). Breast cancer is a multifactorial disease stemming from a complex interplay between environmental, reproductive/endocrine and genetic risk factors. Dissecting the genetic architecture of breast cancer susceptibility is a pivotal step to understand the cascade of molecular events underlying breast carcinogenesis, which ultimately could lead to better preventive and therapeutic strategies according to the precision medicine principles (2).

Familial aggregation of breast cancer (which occurs in about $10 \%$ of cases) has led to family-based linkage analysis and positional cloning studies demonstrating that rare $(<1 \%)$ germline DNA variation in high to moderate penetrance cancer predisposition genes - such as BRCA1, BRCA2, PTEN, CHEK2, ATM, BRIP1 and PALB2 - accounts for about $15-20 \%$ of the familial risk of this disease $(3,4)$. The residual heritability for breast cancer is believed to be sustained by a polygenic model according to the common disease/common variant hypothesis. Subsequent case-control studies based on the candidate gene approach (also known as hypothesis testing approach) have identified some common germline variants (mainly single nucleotide polymorphisms, SNPs) linked to breast cancer risk, though the evidence quality is often low mainly because of small sample size and result heterogeneity $(5,6)$. More recently, the completion of the Human Genome Project and the implementation of genome-wide association studies (GWAS) - based on a hypothesis generating (also known as data driven) approach and testing hundreds of thousands of SNPS at a time - has greatly accelerated the pace of discovery of low penetrance variants linked to the risk of many diseases, including several cancer types (7).

To date, tens of GWAS dedicated to breast cancer have been published, and many single SNPs have been associated with the risk of this malignancy $(3,6)$. This has led to an overwhelming wealth of data which are often difficult to manage by the single reader, in part because most susceptibility loci are intergenic (and thus 
are linked neither to an obvious gene nor to an obvious functional effect), which hinders a straightforward biological interpretation typical of candidate gene studies.

With the present work we intended to systematically review breast cancer GWAS findings in order to provide readers with the first publicly available knowledge-base dedicated to the relationship between germline genomic DNA variation and breast cancer risk. According to the above mentioned polygenic model of sporadic tumor inheritance and using modern SNP-to-gene and gene-to-function approaches such as integrative analysis of genomic data $(8,9)$ as well as pathway and network analysis $(10,11)$, we also aimed to suggest a biological interpretation of current findings. In particular, we tried to exploit the available GWAS evidence to comprehensively identify the cell pathways whose germline variation condition the predisposition to breast cancer, with an additional effort to prioritize genes/pathways/networks which could be of special relevance to inform future studies in the fields of both molecular epidemiology and biology of breast cancer. 


\section{Materials and Methods}

We collected GWAS findings on breast cancer risk (along with other genomic data, see below) to identify breast cancer risk associated SNPs, which were then linked to breast cancer predisposition genes (BCPGs): the data from this knowledge-base were used to perform pathway and network analysis. A flowchart of the study design is reported in Figure 1.

\section{Figure 1}

Study design: flow chart of the integrative analysis of genomic data on breast cancer susceptibility 


\section{GWAS Catalog}

PubMed
Original GWAS

GWAS meta-analysis
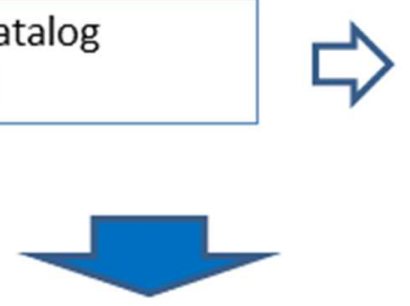

Data filtering

P-value $<1 \times 10 E-06$ MAF $>0.01$
Breast cancer risk associated SNPs
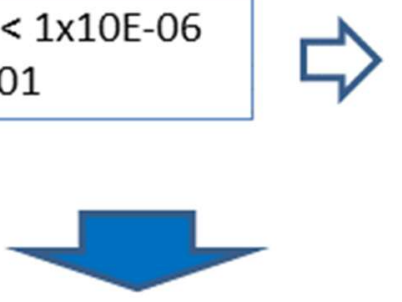

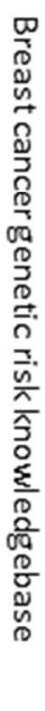
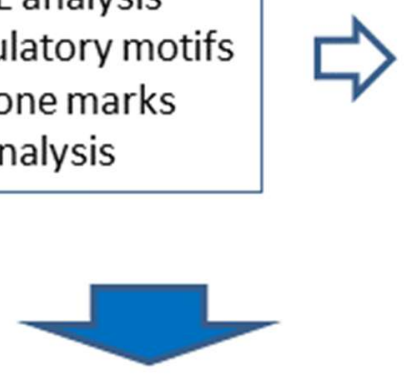

\section{Breast cancer} predisposition genes (BCPGs)

\section{Gene sets}

Based on: all breast cancer susceptibility SNPS, pleiotropic (shared) SNPs, SNPs affectiong transcription factors, SNPs by estrogen receptor status

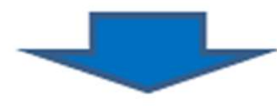




\section{Breast cancer risk associated SNPS}

GWAS addressing the role of germ-line single nucleotide polymorphisms (SNPs) in breast cancer susceptibility were retrieved in the GWAS Catalog repository (7) as well as by performing a systematic review in PubMed (search terms: "breast cancer", "GWAS"). GWAS meta-analyses were also included for data extraction. Searches were updated until the $11^{\text {th }}$ of December 2017.

To be included in the knowledge-base, each SNP had to be associated with breast cancer risk with a nominal P-value lower than 1x10E-06 (genome-wide significance level) and have a minor allele frequency $\geq 1 \%$ in the general population of European ancestry.

\section{$\underline{\text { SNP-to-gene analysis }}$}

Following the principles of integrative analysis of genomic data $(8,9)$, the functional association between a breast cancer risk associated SNP and a gene (hereafter called BCPG) was scored according to three types of information:

A) SNP relationship with gene(s):

[Category 1 - score=2] This applies to within-gene non-synonymous variants (e.g., missense SNPs), variants associated with expression quantitative trait locus (eQTL) data (based on GTex portal database (12)), variants in high linkage disequilibrium (LD) (pairwise $r$-squared $\geq 0.8$ ) with another SNP that is an eQTL hit, or variants in high LD with a within-gene non-synonymous variant;

[Category 2 - score=1] This applies to within-gene synonymous variants, or variants located in a noncoding gene region (e.g., intronic SNPs), or variants in high LD with another within gene SNP (synonymous variant, or variant located in a non-coding gene region);

[Category 3 - score $=0$ ] intergenic and non eQTL hit variants. 
B) SNP relationship with regulatory motifs (i.e., variant potentially affecting the binding of transcription factors based on a library of position weight matrices scored on genomic sequences (13)):

[Category 1 - score=1] variant changing these motifs, or variant in high LD with another SNP changing these motifs;

[Category 2 - score $=0$ ] no change of motif/LD with SNP changing motif.

C) SNP relationship with breast tissue specific promoter/enhancer histone marks (based on data from the Roadmap Epigenomics Project (14)):

[Category 1, score=1] variant co-localization with these marks, or variant in high LD with another SNP co-localising with these marks;

[Category 2, score=0] no co-localization with epigenetic marks/LD with SNP colocalising with epigenetic marks.

The principles underlying the above scoring system are analogous to those employed in well-established functional annotation databases (such as RegulomeDB (15) and HaploReg (16)). However, we added the information deriving from LD analysis (which was performed using the LDLink website (17)), which increases the likelihood of identifying additional functional variants relevant to disease susceptibility. 
bioRxiv preprint doi: https://doi.org/10.1101/279984 - this version posted March 12, 2018. The copyright holder for this preprint (which was

not certified by peer review) is the author/funder, who has granted bioRxiv a license to display the preprint in perpetuity. It is made available under aCC-BY-NC-ND 4.0 International license.

\section{Gene-to-function: pathway and network analysis}

Once BCPGs were identified, we used them to perform pathway and network analysis in order to identify biological functions whose genetic perturbations can predispose to breast cancer development $(10,11)$.

For pathway analysis purposes we utilized gene set enrichment analysis (GSEA) as performed by the EnrichR web server (18). Hypergeometric distribution was used to calculate the statistical significance of gene overlapping (19), followed by correction for multiple hypotheses testing (using the false discovery rate [FDR] method (20)). Only pathways with a FDR $<0.05$ were considered of interest.

Also protein-protein interaction (PPI) networks can be employed to select gene sets. In contrast to pathways, networks are not based on specific biological functions but are built on the basis of both direct (physical) and indirect (genetic) interactions between gene products (proteins). For network analysis, we utilized the STRING 10.5 web server (21). In order to consider only highly reliable information on protein-protein interactions (PPI), we set the interaction score to $\geq 0.7$ (high confidence). The resulting network provides information of the degree of overall connectivity across imputed gene products (as quantified by the ratio between observed and expected interactions [a.k.a. "edges"] between proteins [a.k.a. "nodes"], and formally tested by means of a PPI enrichment test). Then, molecular clusters (subnetworks) can be identified that can be utilized for gene set enrichment analysis (only subnetworks with at least three BCPGs were considered). When the network connectivity is low, the PPI database can be exploited to add first-shell interactors (we chose to add no more than 10 such interactors to avoid data over-interpretation) and then re-run pathway analysis. Ultimately, this data augmentation process increases the likelihood of identifying relevant biological pathways which would be otherwise overlooked when starting with only few BCPGs belonging to a given pathway. 


\section{Other analyses}

Within the frame of network analysis, we searched for so called "hub proteins": these are molecules with a high degree of connectivity with the other network components and thus are likely to play a dominant role in the activity of the network itself (they are also known as "influencers") (22). To this aim, we used the EsyN webtool (23) to calculate the collective influence score, which defined as the product of a node-reduced degree (number of edges minus one) times the sum of the reduced degree of the nodes that are two steps away (a.k.a. radius) (24).

Finally, in order to provide further information beyond the cis effects of included variants (as done in the above analyses), we explored the potential effect in trans of breast cancer associated SNPs. To this aim, we first identified the transcription factors among the putative BCPGs: then, the genes whose expression were regulated by these transcription factors (identified by using the Uniprot (25) and TRRUST (26) repositories) were input in both pathway and network analysis to assess the cellular functions potentially affected by germline variation linked to cancer risk. 


\section{Results}

\section{Breast cancer risk associated SNPS}

We found 38 studies (published between 2007 and 2017) which matched our inclusion criteria (27-64). Of these, 28 were original case-control GWAS (overall enrolling 383,260 patients with breast cancer) and 10 were meta-analyses of previously published case-control GWAS (overall enrolling 239,271 patients with breast cancer) (Supplementary Table 1).

In most studies, patients and controls were of European ancestry ( $71 \%$ among original GWAS; $97 \%$ among GWAS meta-analyses); in the remaining studies, individuals were mainly of Asian ancestry among original GWAS (96\%) and African-American among GWAS meta-analyses (100\%). Only one original study was dedicated to male breast cancer. As regards tumor subtype by estrogen receptor expression, two original GWAS were dedicated to receptor negative and two to receptor positive breast cancer, whereas four GWAS meta-analyses were dedicated to ER negative cases. In the original articles associations were reported (and are reported hereafter in the text) as per-allele odds ratios (ORs).

Overall, 281 SNPs were associated with the risk of breast cancer with a P-value $<10 \mathrm{E}-06$ and a MAF $>0.01$

(Supplementary Table 2); the median minor allele frequency was 0.28 (interquartile range [IQR]: 0.16-0.39); the median OR was 0.93 (IQR: 0.91-0.95) and 1.11 (IQR: 1.06-1.19) for protective and risk alleles, respectively. Chromosome distribution showed an over-representation of chromosome 5 (signals observed: 32; expected: 15; FDR: 0.0003) and chromosome 19 (signals observed: 12; expected: 5; FDR: 0.014).

Linkage disequilibrium (LD) analysis of the 281 SNPs showed that 48 polymorphisms were tagged by one or more other variants (LD r-squared $>0.8$ ), leading to the identification of 233 breast cancer predisposition loci (Supplementary Table 3).

Out of 281 reported SNPs associated with breast cancer risk at a genome-wide significance level, only 34 (12.1\%) were reported by two or more data sources. 
bioRxiv preprint doi: https://doi.org/10.1101/279984; this version posted March 12, 2018. The copyright holder for this preprint (which was not certified by peer review) is the author/funder, who has granted bioRxiv a license to display the preprint in perpetuity. It is made available under aCC-BY-NC-ND 4.0 International license.

Whereas most studies ( $n=21,55.3 \%$ ) enrolled women with unspecified sporadic breast cancer, subgroups were specifically investigated by others: estrogen receptor negative breast cancer $(n=7)$; estrogen receptor positive tumor $(n=2)$; triple negative tumor $(n=1)$; breast cancer in BRCA1/2 mutation carriers (studies, $n=3$ ); early onset breast cancer $(n=1)$; breast cancer in post-menopausal women $(n=1)$; lobular carcinoma $(n=1)$; and breast cancer in males $(n=1)$.

\section{Breast cancer predisposition genes}

The majority of SNPs were located within coding genes ( $n=160,56.9 \%)$. More specifically, SNPs were located in gene $3^{\prime}$-UTR ( $\left.n=7,2.5 \%\right)$, intron $(n=140,49.8 \%)$, exon ( $n=13,4.6 \%$; of these: missense, $n=8$, synonymous, $n=4$ and non-sense [stop gain], $n=1)$. The remaining SNPs were intergenic region ( $n=95,33.8 \%)$ and within non-coding genes ( $n=17,9.2 \%)$. Of note, 6 intergenic SNPs (2.1\%) were in high LD with non-tested SNPs located within a gene and other 8 SNPs $(2.8 \%)$ were in high LD with non-tested missense variants.

As regards eQTL analysis, 107 SNPs (38.1\%) were directly associated with a significant effect on the expression of one or more genes, and 3 SNPs (1.1\%) were in high LD with SNPs with an eQTL effect. A large majority of 229 variants (81.5\%) were associated with changing regulatory motifs, with 43 SNPs (15.3\%) in high LD with those 229 variants, whereas only 9 SNPs had no impact on regulatory motifs. In addition, 79 SNPs (28.1\%) co-localized with promoter/enhancer histone marks, with 107 variants (38.1\%) in high LD with those 79 SNPs, and 95 SNPs (33.8\%) having no such property.

Based on the above information, we associated the 281 SNPs linked with breast cancer risk to 334 genes with a score ranging from 0 to 4 (Supplementary Table 4): SNPs with low (0-1), intermediate (2) and high (3-4) functional score were 30 (10.7\%), 68 (24.2\%), and $183(65.1 \%)$, respectively. In order to exclude genes with low level of evidence of association with breast cancer risk SNPs, we further considered only SNPs with a score equal or greater than 2 ( $n=251)$. With this cut off, we identified 296 putative BCPGs, which were the genes utilized in the following primary analysis. These genes code for known proteins in most cases ( $n=255$, $86.1 \%)$. 
bioRxiv preprint doi: $h t t p s: / /$ doi org/10.1101/279984; this version posted March 12, 2018. The copyright holder for this preprint (which was not certified by peer review) is the author/funder, who has granted bioRxiv a license to display the preprint in perpetuity. It is made available under aCC-BY-NC-ND 4.0 International license.

\section{Pathway and network analysis}

Primary gene set enrichment analysis demonstrated that the 296 BCPGs are enriched in genes involved in apoptotic pathway and peroxisome machinery, as illustrated in Table 1.

Table 1: Pathway analysis of breast cancer predisposition genes (BCPGs). Overlap: number of BCPGs over number of pathway genes. FDR: false discovery rate.

\begin{tabular}{|c|c|c|c|}
\hline \multicolumn{4}{|c|}{ PRIMARY ANALYSIS (296 BCPGs) } \\
\hline Pathway & Overlap & FDR & Genes \\
\hline Apoptosis & $9 / 140$ & 0.04330 & PIDD1;DFFA;BCL2L11;CASP8;TNFSF10;ITPR1;PIK3R3;CTSW;CFLAR \\
\hline Peroxisomal importomer & $18 / 82$ & $<0.00001$ & $\begin{array}{l}\text { DCLRE1B;TCF7L2;DFFA;CDKN2B;STXBP4;PDE4D;MKL1;EBF1;LSP1;PEX14 } \\
\text {;RAD51B; } \\
\text { BABAM1;TOX3;ADAM29; RALY;MDM4;LGR6;CASC16 }\end{array}$ \\
\hline \multicolumn{4}{|c|}{ FIRST-SHELL AUGMENTATION (296 BCPGs + 10 interactors) } \\
\hline Pathway & Overlap & FDR & Genes \\
\hline Prostate cancer & $6 / 89$ & 0.04198 & TCF7L2;CCND1;CCNE1;PIK3R3;FGFR2;CREB5 \\
\hline Apoptosis & $8 / 140$ & 0.02506 & DFFA;BCL2L11;CASP8;TNFSF10;ITPR1;PIK3R3;CTSW;CFLAR \\
\hline FoxO signaling & $8 / 133$ & 0.02373 & CDKN2B;BCL2L11;CCND1;IRS1;TNFSF10;PIK3R3;FBXO32;TGFBR2 \\
\hline Ribosome & $10 / 137$ & 0.00261 & RPS16;RPS15A;RPS29;RPLP1;RPS3;RPLP2;RPS2;MRPL34;RPS13;RPS23 \\
\hline $\begin{array}{l}\text { Non-alcoholic fatty liver } \\
\text { disease }\end{array}$ & $9 / 151$ & 0.01670 & ITCH;NDUFA13;BCL2L11;CASP8;IRS1;NDUFB3;NDUFA2;PIK3R3;COX6A1 \\
\hline \multicolumn{4}{|c|}{ SMALL SUBNETWORK (5 BCPGs) } \\
\hline Pathway & Overlap & FDR & Genes \\
\hline Translation Initiation & $3 / 114$ & 0.00001 & RPLP2;EIF2S2;RPS23 \\
\hline Ribosome formation & $3 / 180$ & 0.00003 & RPLP2;WDR43;RPS23 \\
\hline \multicolumn{4}{|c|}{ LARGE SUBNETWORK (59 BCPGs) } \\
\hline Pathway & Overlap & FDR & Genes \\
\hline Pathways in cancer & $11 / 397$ & $<0.00001$ & $\begin{array}{l}\text { TCF7L2;CDKN2B;CASP8;CCND1;CCNE1;ADCY3;LPAR2;PIK3R3;BRCA2;FG } \\
\text { FR2;TGFBR2 }\end{array}$ \\
\hline Pancreatic cancer & $4 / 66$ & 0.00057 & CCND1;PIK3R3;BRCA2;TGFBR2 \\
\hline Prostate cancer & $6 / 89$ & $<0.00001$ & TCF7L2;CCND1;CCNE1;PIK3R3;FGFR2;CREB5 \\
\hline Colorectal cancer & $4 / 62$ & 0.00049 & TCF7L2;CCND1;PIK3R3;TGFBR2 \\
\hline Small cell lung cancer & $4 / 86$ & 0.00118 & CDKN2B;CCND1;CCNE1;PIK3R3 \\
\hline Endometrial cancer & $3 / 52$ & 0.00258 & TCF7L2;CCND1;PIK3R3 \\
\hline Acute myeloid leukemia & $3 / 57$ & 0.00312 & TCF7L2;CCND1;PIK3R3 \\
\hline Chronic myeloid leukemia & $3 / 73$ & 0.00520 & CCND1;PIK3R3;TGFBR2 \\
\hline Proteoglycans in cancer & $5 / 203$ & 0.00219 & PPP1CB;CCND1;ERBB4;PIK3R3;ESR1 \\
\hline
\end{tabular}


bioRxiv preprint doi: https://doi org/10.1101/279984; this version posted March 12, 2018. The copyright holder for this preprint (which was not certified by peer review) is the author/funder, who has granted bioRxiv a license to display the preprint in perpetuity. It is made available under aCC-BY-NC-ND 4.0 International license.

\begin{tabular}{|c|c|c|c|}
\hline MicroRNAs in cancer & $4 / 297$ & 0.03057 & BCL2L11;CCND1;CCNE1;IRS1 \\
\hline FoxO signaling & $8 / 133$ & $<0.00001$ & CDKN2B;BCL2L11;CCND1;IRS1;TNFSF10;PIK3R3;FBXO32;TGFBR2 \\
\hline PI3K-Akt signaling & $10 / 341$ & $<0.00001$ & $\begin{array}{l}\text { EFNA1;COL1A2;BCL2L11;CCND1;CCNE1;IRS1;LPAR2;PIK3R3;FGFR2;CRE } \\
\text { B5 }\end{array}$ \\
\hline p53 signaling & $3 / 69$ & 0.00481 & CASP8;CCND1;CCNE1 \\
\hline cAMP signaling & $6 / 199$ & 0.00048 & PPP1CB;GIPR;PDE4D;ADCY3;PIK3R3;CREB5 \\
\hline Rap1 signaling & $5 / 211$ & 0.00249 & EFNA1;ADCY3;LPAR2;PIK3R3;FGFR2 \\
\hline AMPK signaling & $4 / 124$ & 0.00258 & CCND1;IRS1;PIK3R3;CREB5 \\
\hline Hippo signaling & $4 / 153$ & 0.00481 & PPP1CB;TCF7L2;CCND1;TGFBR2 \\
\hline MAPK signaling & $4 / 255$ & 0.02078 & MAP3K1;TAB2;FGFR2;TGFBR2 \\
\hline Phospholipase D signaling & $3 / 144$ & 0.02410 & ADCY3;LPAR2;PIK3R3 \\
\hline TNF signaling & $6 / 110$ & 0.00002 & ITCH;CASP8;PIK3R3;TAB2;CFLAR;CREB5 \\
\hline Longevity regulation & $4 / 94$ & 0.00147 & IRS1;ADCY3;PIK3R3;CREB5 \\
\hline Apoptosis & $5 / 140$ & 0.00065 & BCL2L11;CASP8;TNFSF10;PIK3R3;CFLAR \\
\hline Cell cycle & $4 / 124$ & 0.00258 & CDKN2B;CCND1;CCNE1;ORC2 \\
\hline Viral carcinogenesis & $6 / 205$ & 0.00049 & CDKN2B;CASP8;CCND1;CCNE1;PIK3R3;CREB5 \\
\hline Hepatitis B & $6 / 146$ & 0.00010 & MAP3K1;CASP8;CCND1;CCNE1;PIK3R3;CREB5 \\
\hline Measles & $5 / 136$ & 0.00061 & CCND1;CCNE1;TNFSF10;PIK3R3;TAB2 \\
\hline HTLV-I infection & $7 / 258$ & 0.00022 & CDKN2B;MAP3K1;TERT;CCND1;ADCY3;PIK3R3;TGFBR2 \\
\hline Herpes simplex infection & $3 / 185$ & 0.04043 & PPP1CB;CASP8;TAB2 \\
\hline Estrogen signaling & $4 / 99$ & 0.00170 & ADCY3;PIK3R3;ESR1;CREB5 \\
\hline $\begin{array}{l}\text { Thyroid hormone } \\
\text { signaling }\end{array}$ & $4 / 118$ & 0.00249 & NCOA1;CCND1;PIK3R3;ESR1 \\
\hline Oxytocin signaling & $4 / 158$ & 0.00511 & PPP1CB;CCND1;ADCY3;PIK3R3 \\
\hline Prolactin signaling & $3 / 72$ & 0.00513 & CCND1;PIK3R3;ESR1 \\
\hline Focal adhesion & $4 / 202$ & 0.01145 & PPP1CB;COL1A2;CCND1;PIK3R3 \\
\hline Toll-like receptor signaling & $3 / 106$ & 0.01327 & CASP8;PIK3R3;TAB2 \\
\hline Endocytosis & $4 / 259$ & 0.02105 & ITCH;ERBB4;FGFR2;TGFBR2 \\
\hline
\end{tabular}


Network analysis suggested that BCPGs protein products did not have more interactions among themselves than expected for a random protein set of equal size drawn from the proteome (observed edges: 98; expected edges: 83; PPI enrichment test P-value: 0.0527), indicating that these proteins are not remarkably biologically connected as a group. When 10 first-shell interactors were added to the network, ribosome proteins were then included in the enrichment list (Table 1).

Network analysis also enabled us to identify one large $(n=59)$ and one small $(n=5)$ subnetwork (Figure 2): the former was enriched in several cancer-related pathways, including apoptosis and estrogen receptor signaling, whereas the latter was enriched in ribosome machinery components (see Table 1). Finally, influence analysis of the large subnetwork identified estrogen receptor 1 (ESR1) as the most influential protein (Suppementary Table 5).

\section{Figure 2}

Network analysis: network plot of protein-protein interactions regarding the products of the putative breast cancer predisposition genes identified through the integrative analysis of GWAS data 
bioRxiv preprint doi: https://doi org/10.1101/279984; this version posted March 12, 2018. The copyright holder for this preprint (which was not certified by peer review) is the author/funder, who has granted bioRxiv a license to display the preprint in perpetuity. It is made available under aCC-BY-NC-ND 4.0 International license.

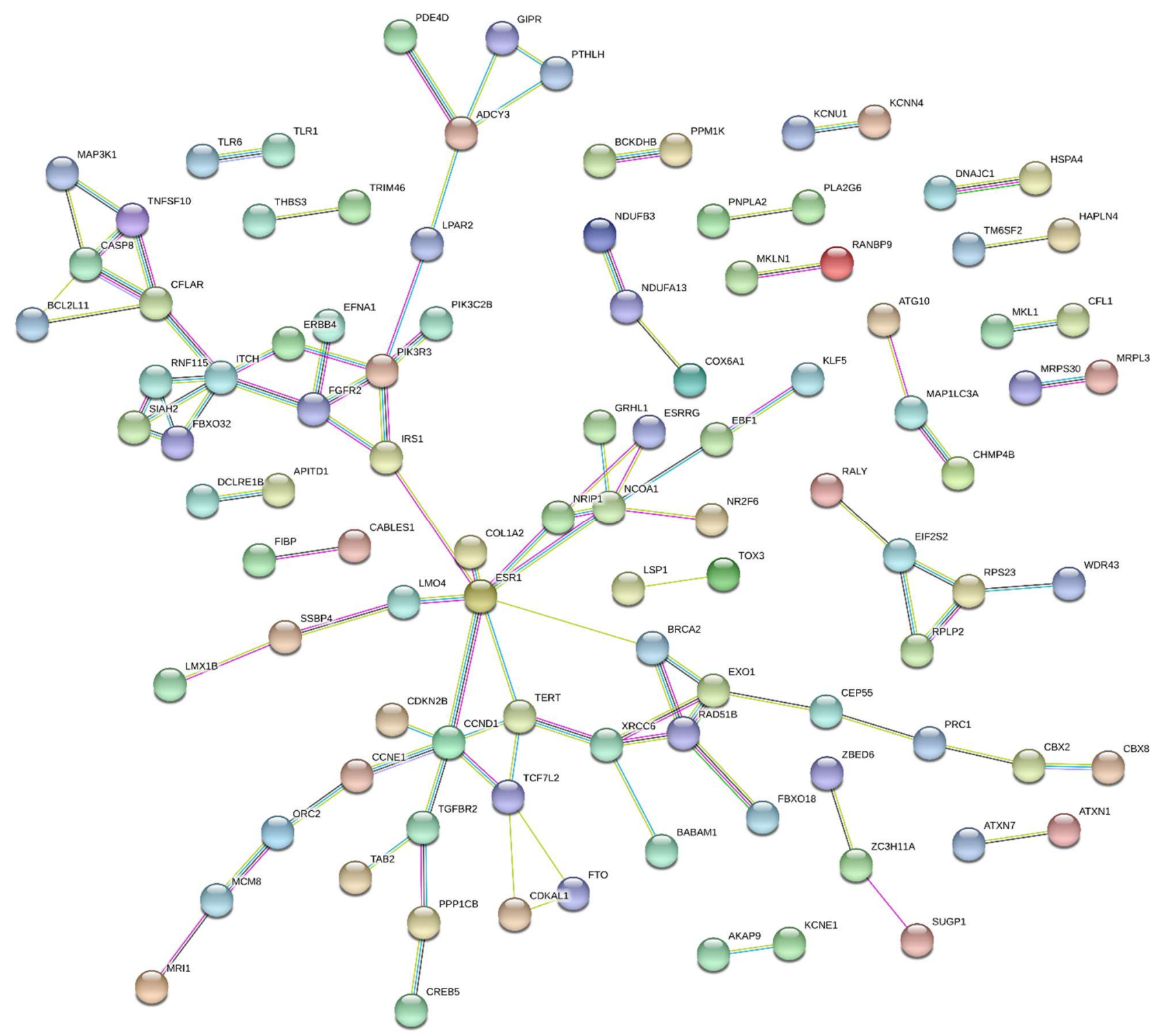




\section{$\underline{\text { Subgroup analysis }}$}

In a first subgroup analysis we considered the BCPGs encoding transcription factors: there were 36 such genes (Figure 3), which represent $12.2 \%$ of the BCPGs identified in this work, a figure only slightly higher than expected (10\%). These transcription factors target 252 genes (Figure 3), with nine also being BCPGs (AHRR, BRCA2, CCND1, CDKN2B, ESR1, FOXP1, FTO, LPAR2, TERT).

\section{Figure 3}

Breast cancer susceptibility regulons: targets of transcription factors whose germline variation is associated with breast cancer risk 
bioRxiv preprint doi: https://doi.org/10.1101/279984; this version posted March 12, 2018. The copyright holder for this preprint (which was not certified by peer review) is the author/funder, who has granted bioRxiv a license to display the preprint in perpetuity. It is made available under aCC-BY-NC-ND 4.0 International license.

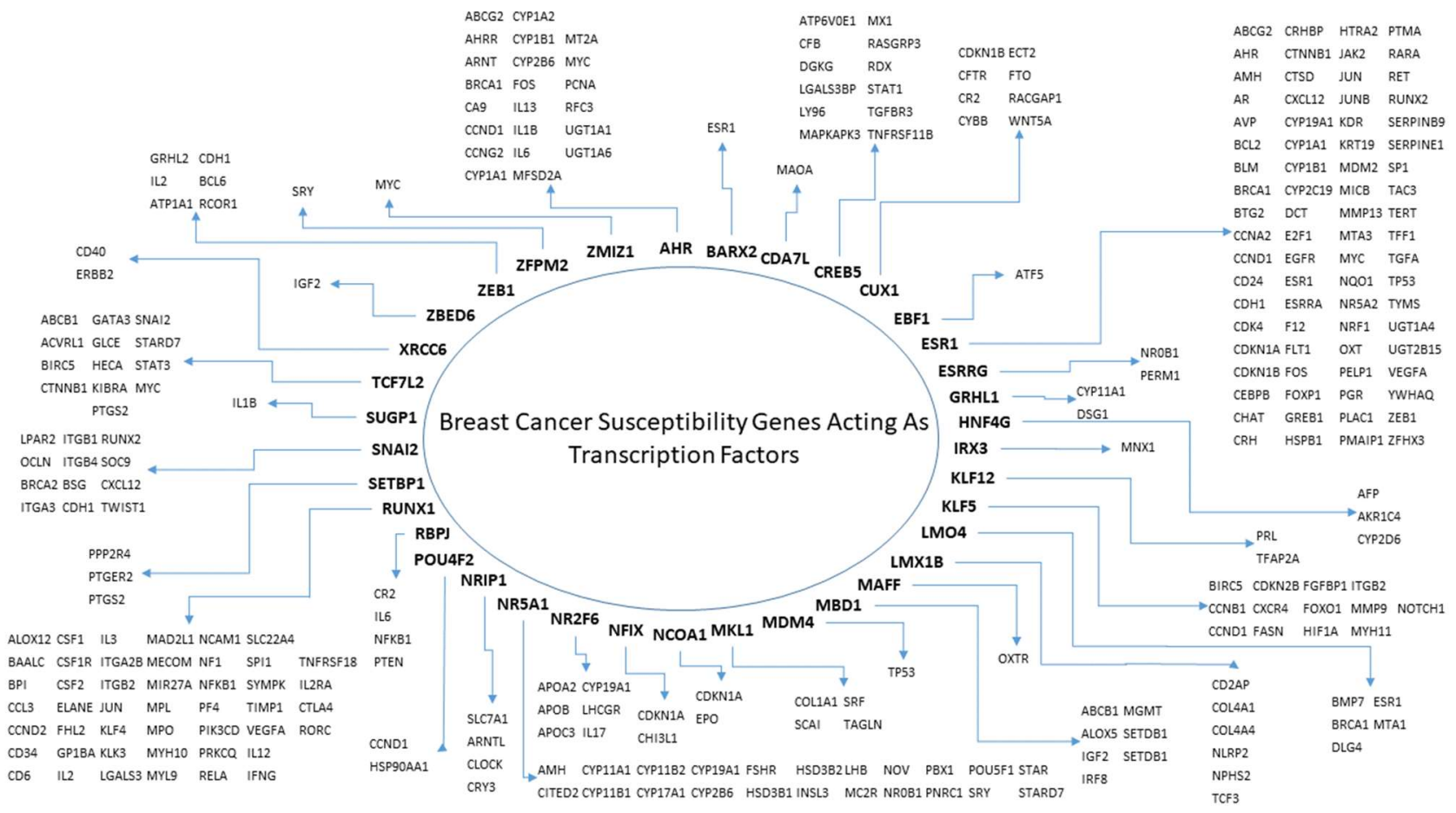


bioRxiv preprint doi: https://doi.org/10.1101/279984; this version posted March 12, 2018. The copyright holder for this preprint (which was not certified by peer review) is the author/funder, who has granted bioRxiv a license to display the preprint in perpetuity. It is made available under aCC-BY-NC-ND 4.0 International license.

Pathway analysis of the 252 targets demonstrated a significant enrichment in many cancer-related pathways, including those involved in the pathogenesis of different tumor types (mainly but not only carcinomas), cell cycle and apoptosis, multiple signaling pathways (such as p53, PI3K-Akt, Wnt, Hippo, Mapk, ErbB, HIF-1 and VEGF), hormone pathways (including sex hormones), immunity (with special regard to anti-viral immune response), and cell adhesion (Table 2).

Table 2: Pathway analysis of breast cancer predisposition genes (BCPGs) encoding transcription factors. Overlap: number of BCPGs over number of pathway genes. FDR: false discovery rate.

\begin{tabular}{|c|c|c|c|}
\hline Pathways & Overlap & FDR & Genes \\
\hline Pathways in cancer & $51 / 397$ & $<0.00001$ & $\begin{array}{l}\text { RET;ITGB1;CDKN1A;SPI1;CDKN1B;ITGA2B;PTEN;PIK3CD;BRCA2;MECOM;CCND1; } \\
\text { CDH1;MYC;HSP90AA1;ITGA3;WNT5A;ARNT;FOS;MMP9;AR;COL4A1;COL4A4; } \\
\text { RARA;BIRC5;TP53;CSF1R;PTGER2;LPAR2;TGFA;CXCR4;KLK3;PTGS2;HIF1A;EGFR; } \\
\text { FOXO1;RELA;RASGRP3;ERBB2;E2F1;IUN;CDKN2B;STAT1;STAT3;NFKB1;VEGFA; } \\
\text { IL6;CXCL12;CDK4;MDM2;BCL2;CTNNB1 }\end{array}$ \\
\hline Prostate cancer & $19 / 89$ & $<0.00001$ & $\begin{array}{l}\text { HSP90AA1;CDKN1A;CDKN1B;PTEN;TGFA;PIK3CD;KLK3;RELA;EGFR;FOXO1;NFKB1; } \\
\text { AR;CCND1;ERBB2;BCL2;E2F1;MDM2;CTNNB1;TP53 }\end{array}$ \\
\hline Small cell lung cancer & $18 / 86$ & $<0.00001$ & $\begin{array}{l}\text { ITGB1;CDKN2B;CDKN1B;ITGA3;ITGA2B;PTEN;PIK3CD;PTGS2;RELA;NFKB1;CCND1; } \\
\text { CDK4;COL4A1;MYC;COL4A4;BCL2;E2F1;TP53 }\end{array}$ \\
\hline Non-small cell lung cancer & $8 / 56$ & $<0.00001$ & CCND1;CDK4;ERBB2;E2F1;TGFA;PIK3CD;TP53;EGFR \\
\hline Pancreatic cancer & $14 / 66$ & $<0.00001$ & $\begin{array}{l}\text { STAT1;STAT3;TGFA;PIK3CD;BRCA2;RELA;EGFR;NFKB1;VEGFA;CCND1;CDK4;ERBB2; } \\
\text { E2F1;TP53 }\end{array}$ \\
\hline Bladder cancer & $12 / 41$ & $<0.00001$ & CDKN1A;CCND1;CDK4;CDH1;MYC;ERBB2;MDM2;E2F1;MMP9;TP53;EGFR;VEGFA \\
\hline Chronic myeloid leukemia & $12 / 73$ & $<0.00001$ & $\begin{array}{l}\text { CDKN1A;CDKN1B;MECOM;CCND1;CDK4;MYC;E2F1;MDM2;PIK3CD;TP53;RELA; } \\
\text { NFKB1 }\end{array}$ \\
\hline Glioma & $10 / 65$ & $<0.00001$ & CDKN1A;CCND1;CDK4;E2F1;MDM2;PTEN;TGFA;PIK3CD;TP53;EGFR \\
\hline Melanoma & $10 / 71$ & $<0.00001$ & CDKN1A;CCND1;CDK4;CDH1;E2F1;MDM2;PTEN;PIK3CD;TP53;EGFR \\
\hline Endometrial cancer & $9 / 52$ & $<0.00001$ & CCND1;CDH1;MYC;ERBB2;PTEN;CTNNB1;PIK3CD;TP53;EGFR \\
\hline Colorectal cancer & $9 / 62$ & $<0.00001$ & JUN;CCND1;MYC;BCL2;BIRC5;CTNNB1;PIK3CD;FOS;TP53 \\
\hline Renal cell carcinoma & $6 / 66$ & 0.00052 & JUN;TGFA;ARNT;PIK3CD;HIF1A;VEGFA \\
\hline Acute myeloid leukemia & $8 / 57$ & $<0.00001$ & SPI1;CCND1;MYC;STAT3;RARA;PIK3CD;RELA;NFKB1 \\
\hline PI3K-Akt signaling & $37 / 341$ & $<0.00001$ & $\begin{array}{l}\text { ITGB1;CSF1R;CDKN1A;CDKN1B;FLT1;CSF1;EPO;ITGB4;ITGA2B;PTEN;LPAR2;PIK3CD; } \\
\text { PRL;BRCA1;RELA;EGFR;CCND2;CCND1;YWHAQ;MYC;KDR;JAK2;HSP90AA1;ITGA3; } \\
\text { NFKB1;IL2;VEGFA;COL1A1;IL3;IL6;COL4A1;CDK4;COL4A4;IL2RA;MDM2;BCL2;TP53 }\end{array}$ \\
\hline MAPK signaling & $14 / 255$ & 0.00001 & $\begin{array}{l}\text { JUN;SRF;HSPB1;FOS;RELA;EGFR;NFKB1;RASGRP3;MECOM;MAPKAPK3;MYC;IL1B; } \\
\text { NF1;TP53 }\end{array}$ \\
\hline ErbB signaling & $8 / 87$ & 0.00004 & JUN;CDKN1A;CDKN1B;MYC;ERBB2;TGFA;PIK3CD;EGFR \\
\hline Ras signaling & $11 / 227$ & 0.00043 & CSF1R;FLT1;CSF1;KDR;NF1;PIK3CD;RELA;EGFR;NFKB1;VEGFA;RASGRP3 \\
\hline p53 signaling & $11 / 69$ & $<0.00001$ & CDKN1A;CCNB1;CCND2;CCND1;CDK4;CCNG2;SERPINE1;MDM2;PTEN;PMAIP1;TP53 \\
\hline FoxO signaling & $15 / 133$ & $<0.00001$ & $\begin{array}{l}\text { CDKN2B;CDKN1A;CDKN1B;STAT3;PTEN;PIK3CD;EGFR;FOXO1;CCNB1;IL6;CCND2; } \\
\text { CCND1;BCL6;CCNG2;MDM2 }\end{array}$ \\
\hline cAMP signaling & $13 / 199$ & $<0.00001$ & OXTR;JUN;PTGER2;PIK3CD;FOS;ATP1A1;RELA;NFKB1;MC2R;FSHR;AMH;MYL9;CFTR \\
\hline Rap1 signaling & $14 / 211$ & $<0.00001$ & ITGB1;CSF1R;FLT1;CSF1;ITGA2B;ITGB2;LPAR2;PIK3CD;EGFR;VEGFA;RASGRP3;CDH1; \\
\hline
\end{tabular}


bioRxiv preprint doi: https://doi.org/10.1101/279984; this version posted March 12, 2018. The copyright holder for this preprint (which was not certified by peer review) is the author/funder, who has granted bioRxiv a license to display the preprint in perpetuity. It is made available under aCC-BY-NC-ND 4.0 International license.

\begin{tabular}{|c|c|c|c|}
\hline & & & KDR;CTNNB1 \\
\hline Chemokine signaling & $10 / 187$ & 0.00039 & CXCL12;STAT1;STAT3;CCL3;PIK3CD;CXCR4;JAK2;RELA;NFKB1;PF4 \\
\hline Jak-STAT signaling & 19/158 & $<0.00001$ & $\begin{array}{l}\text { CDKN1A;CSF2;EPO;STAT1;IL13;STAT3;MPL;PIK3CD;PRL;IL2;IL3;IL6;CCND2;CCND1; } \\
\text { IFNG;MYC;IL2RA;BCL2;JAK2 }\end{array}$ \\
\hline NF-kappa B signaling & 9/93 & 0.00001 & CD40;CXCL12;IL1B;BCL2;LY96;PRKCQ;PTGS2;RELA;NFKB1 \\
\hline Hippo signaling & $15 / 153$ & $<0.00001$ & $\begin{array}{l}\text { WNT5A;SERPINE1;ITGB2;AFP;BMP7;CCND2;CCND1;YWHAQ;CDH1;DLG4;MYC;BIRC5; } \\
\text { SNAI2;CTNNB1;AMH }\end{array}$ \\
\hline Wnt signaling & $7 / 142$ & 0.00496 & JUN;CCND2;CCND1;MYC;WNT5A;CTNNB1;TP53 \\
\hline HIF-1 signaling & $19 / 103$ & $<0.00001$ & $\begin{array}{l}\text { CDKN1A;FLT1;CDKN1B;EPO;SERPINE1;STAT3;CYBB;ARNT;PIK3CD;HIF1A;RELA;EGFR; } \\
\text { NFKB1;VEGFA;IL6;IFNG;ERBB2;BCL2;TIMP1 }\end{array}$ \\
\hline VEGF signaling & $6 / 61$ & 0.00034 & MAPKAPK3;KDR;HSPB1;PIK3CD;PTGS2;VEGFA \\
\hline TNF signaling & $13 / 110$ & $<0.00001$ & JUN;CEBPB;CSF2;CSF1;PIK3CD;FOS;PTGS2;MMP9;RELA;NFKB1;IL6;IL1B;JUNB \\
\hline Apoptosis & $12 / 140$ & $<0.00001$ & IL3;JUN;BCL2;BIRC5;HTRA2;PMAIP1;PIK3CD;FOS;TP53;CTSD;RELA;NFKB1 \\
\hline Cell cycle & $15 / 124$ & $<0.00001$ & $\begin{array}{l}\text { CDKN2B;CDKN1A;PCNA;CDKN1B;CCNA2;CCNB1;CCND2;CCND1;YWHAQ;CDK4;MYC; } \\
\text { E2F1;MDM2;TP53;MAD2L1 }\end{array}$ \\
\hline Estrogen signaling & $8 / 99$ & 0.00012 & HSP90AA1;JUN;SP1;PIK3CD;FOS;ESR1;MMP9;EGFR \\
\hline $\begin{array}{l}\text { Steroid hormone } \\
\text { biosynthesis }\end{array}$ & $15 / 58$ & $<0.00001$ & $\begin{array}{l}\text { HSD3B2;UGT1A1;HSD3B1;UGT2B15;AKR1C4;CYP19A1;CYP17A1;CYP11B2;CYP11A1; } \\
\text { CYP11B1;CYP1A2;CYP1A1; } \\
\text { CYP1B1;UGT1A4;UGT1A6 }\end{array}$ \\
\hline Prolactin signaling & $14 / 72$ & $<0.00001$ & $\begin{array}{l}\text { LHCGR;STAT1;STAT3;LHB;PIK3CD;PRL;FOS;ESR1;RELA;NFKB1;CYP17A1;CCND2;CCND1; } \\
\text { JAK2 }\end{array}$ \\
\hline Thyroid hormone signaling & $12 / 118$ & $<0.00001$ & NOTCH1;CCND1;STAT1;MYC;MDM2;CTNNB1;PIK3CD;ATP1A1;TP53;ESR1;HIF1A;FOXO1 \\
\hline Oxytocin signaling pathway & $10 / 158$ & 0.00011 & OXTR;JUN;CDKN1A;CCND1;PIK3CD;OXT;FOS;PTGS2;MYL9;EGFR \\
\hline T cell receptor signaling & $11 / 104$ & $<0.00001$ & JUN;CSF2;IFNG;CDK4;CTLA4;PIK3CD;PRKCQ;FOS;RELA;NFKB1;IL2 \\
\hline Toll-like receptor signaling & $11 / 106$ & $<0.00001$ & JUN;IL6;CD40;STAT1;IL1B;CCL3;LY96;PIK3CD;FOS;RELA;NFKB1 \\
\hline $\begin{array}{l}\text { Cytokine-cytokine receptor } \\
\text { interaction }\end{array}$ & $26 / 265$ & $<0.00001$ & $\begin{array}{l}\text { CSF1R;CD40;CSF2;FLT1;CSF1;EPO;MPL;CXCR4;TNFRSF11B;PRL;EGFR;KDR;CCL3;AMH; } \\
\text { TNFRSF18;IL13;BMP7;IL2; } \\
\text { VEGFA;IL3;IL6;CXCL12;IFNG;IL1B;IL2RA;PF4 }\end{array}$ \\
\hline $\begin{array}{l}\text { Leukocyte transendothelial } \\
\text { migration }\end{array}$ & $10 / 118$ & $<0.00001$ & ITGB1;OCLN;CXCL12;ITGB2;CYBB;CTNNB1;PIK3CD;CXCR4;MYL9;MMP9 \\
\hline $\begin{array}{l}\text { Regulation of actin } \\
\text { cytoskeleton }\end{array}$ & $10 / 214$ & 0.00107 & ITGB1;ITGA3;ITGB4;RDX;ITGA2B;ITGB2;PIK3CD;MYL9;MYH10;EGFR \\
\hline Hepatitis B & $22 / 146$ & $<0.00001$ & $\begin{array}{l}\text { JUN;CDKN1A;PCNA;CDKN1B;STAT1;STAT3;PTEN;PIK3CD;FOS;MMP9;RELA;NFKB1; } \\
\text { CCNA2;IL6;CCND1;YWHAQ;CDK4;MYC;E2F1;BCL2;BIRC5;TP53 }\end{array}$ \\
\hline HTLV-I infection & $27 / 258$ & $<0.00001$ & $\begin{array}{l}\text { CD40;CDKN1A;SPI1;PCNA;CSF2;SRF;ITGB2;PIK3CD;RELA;CCND2;TERT;CCND1;MYC; } \\
\text { E2F1;JUN;CDKN2B;WNT5A;FOS;NFKB1;IL2;IL6;CDK4;IL2RA;CTNNB1;TCF3;TP53; } \\
\text { MAD2L1 }\end{array}$ \\
\hline Measles & $19 / 136$ & $<0.00001$ & $\begin{array}{l}\text { CDKN1B;STAT1;MX1;IL13;STAT3;PIK3CD;RELA;NFKB1;IL2;IL6;CCND2;CCND1;IFNG; } \\
\text { CDK4;IL1B;IL2RA;PRKCQ;JAK2;TP53 }\end{array}$ \\
\hline Herpes simplex infection & $12 / 185$ & 0.00001 & JUN;IL6;IFNG;STAT1;IL1B;FOS;JAK2;TP53;CLOCK;RELA;NFKB1;ARNTL \\
\hline Epstein-Barr virus infection & $19 / 202$ & $<0.00001$ & $\begin{array}{l}\text { JUN;CD40;CDKN1A;CR2;SPI1;CDKN1B;STAT3;HSPB1;PIK3CD;RELA;NFKB1;CCNA2; } \\
\text { IFNG;YWHAQ;MYC;MDM2;BCL2;TP53;PTMA }\end{array}$ \\
\hline Influenza A & $10 / 175$ & 0.00023 & JUN;IL6;IFNG;STAT1;IL1B;MX1;PIK3CD;JAK2;RELA;NFKB1 \\
\hline Hepatitis C & $9 / 133$ & 0.00015 & OCLN;CDKN1A;STAT1;STAT3;PIK3CD;TP53;RELA;EGFR;NFKB1 \\
\hline Viral carcinogenesis & $17 / 205$ & $<0.00001$ & $\begin{array}{l}\text { JUN;CDKN2B;CDKN1A;CDKN1B;SRF;STAT3;PIK3CD;RELA;NFKB1;CCNA2;CCND2; } \\
\text { CCND1;YWHAQ;CDK4;MDM2;PMAIP1;TP53 }\end{array}$ \\
\hline Chemical carcinogenesis & $10 / 82$ & $<0.00001$ & UGT1A1;CYP1A2;UGT2B15;CYP1A1;CYP1B1;ARNT;UGT1A4;CYP2C19;PTGS2;UGT1A6 \\
\hline $\begin{array}{l}\text { Drug } \\
\text { metabolism_cytochrome } \\
\text { P450 }\end{array}$ & $9 / 69$ & $<0.00001$ & CYP2B6;UGT1A1;MAOA;CYP2D6;CYP1A2;UGT2B15;UGT1A4;CYP2C19;UGT1A6 \\
\hline $\begin{array}{l}\text { Xenobiotics } \\
\text { metabolism_cytochrome } \\
\text { P450 }\end{array}$ & $9 / 73$ & $<0.00001$ & CYP2B6;UGT1A1;CYP2D6;CYP1A2;UGT2B15;CYP1A1;CYP1B1;UGT1A4;UGT1A6 \\
\hline Insulin resistance & $9 / 109$ & 0.00003 & IL6;PPP2R4;STAT3;PTEN;PIK3CD;PRKCQ;RELA;FOXO1;NFKB1 \\
\hline $\begin{array}{l}\text { Choline metabolism in } \\
\text { cancer }\end{array}$ & $8 / 101$ & 0.00013 & DGKG;SLC22A4;JUN;SP1;PIK3CD;FOS;HIF1A;EGFR \\
\hline Retinol metabolism & $7 / 65$ & 0.00005 & CYP2B6;UGT1A1;CYP1A2;UGT2B15;CYP1A1;UGT1A4;UGT1A6 \\
\hline $\begin{array}{l}\text { Central carbon metabolism } \\
\text { in cancer }\end{array}$ & $8 / 67$ & $<0.00001$ & RET;MYC;ERBB2;PTEN;PIK3CD;TP53;HIF1A;EGFR \\
\hline Focal adhesion & $20 / 202$ & $<0.00001$ & ITGB1;JUN;FLT1;ITGA3;ITGB4;ITGA2B;PTEN;PIK3CD;EGFR;VEGFA;COL1A1;CCND2; \\
\hline
\end{tabular}


bioRxiv preprint doi: https://doi.org/10.1101/279984; this version posted March 12, 2018. The copyright holder for this preprint (which was not certified by peer review) is the author/funder, who has granted bioRxiv a license to display the preprint in perpetuity. It is made available under aCC-BY-NC-ND 4.0 International license.

\begin{tabular}{|l|l|l|l|} 
& & & CCND1;COL4A1;COL4A4;ERBB2;KDR;BCL2;CTNNB1;MYL9 \\
\hline $\begin{array}{l}\text { Cell adhesion molecules } \\
\text { (CAMs) }\end{array}$ & $9 / 142$ & 0.00024 & ITGB1;OCLN;CD40;CD6;CDH1;ITGB2;CTLA4;NCAM1;CD34 \\
\hline Adherens junction & $5 / 74$ & 0.00540 & CDH1;ERBB2;SNAI2;CTNNB1;EGFR \\
\hline Tight junction & $9 / 139$ & 0.00021 & OCLN;CDK4;PTEN;SYMPK;CTNNB1;MYH11;PRKCQ;MYL9;MYH10 \\
\hline ECM-receptor interaction & $8 / 82$ & 0.00003 & COL1A1;ITGB1;ITGA3;COL4A1;ITGB4;COL4A4;ITGA2B;GP1BA \\
\hline $\begin{array}{l}\text { Transcriptional } \\
\text { misregulation in cancer }\end{array}$ & $27 / 180$ & $<0.00001$ & $\begin{array}{l}\text { CSF1R;CD40;CEBPB;CDKN1A;CSF2;SPI1;FLT1;CDKN1B;MPO;RELA;FOXO1;CCND2; } \\
\text { MYC;ELANE;MMP9;RUNX2;NFKB1;PBX1;IL3;IL6;ZEB1;BCL6;SP1;MDM2;RARA; } \\
\text { TCF3;TP53 }\end{array}$ \\
\hline MicroRNAs in cancer & $26 / 297$ & $<0.00001$ & $\begin{array}{l}\text { CDKN1A;CDKN1B;NOTCH1;ABCB1;PTEN;BRCA1;PTGS2;SLC7A1;EGFR;CCND2; } \\
\text { CCND1;MYC;ERBB2;E2F1;CYP1B1;RDX;STAT3;MIR27A;MMP9;NFKB1;VEGFA; } \\
\text { FOXP1;ZEB1;MDM2;BCL2;TP53 }\end{array}$ \\
\hline $\begin{array}{l}\text { Signaling pathways of stem } \\
\text { cells }\end{array}$ & $11 / 142$ & $<0.00001$ & \begin{tabular}{l} 
ZFHX3;SETDB1;MYC;WNT5A;STAT3;CTNNB1;PIK3CD;TCF3;KLF4;JAK2;POU5F1 \\
\hline Proteoglycans in cancer
\end{tabular} \\
\hline
\end{tabular}


bioRxiv preprint doi: https://doi.org/10.1101/279984; this version posted March 12, 2018. The copyright holder for this preprint (which was not certified by peer review) is the author/funder, who has granted bioRxiv a license to display the preprint in perpetuity. It is made available under aCC-BY-NC-ND 4.0 International license.

Network analysis revealed a very high degree of connectivity across these target genes (observed edges: 3105; expected: 1104; PPI enrichment p-value: <10E-16); influence analysis showed that the top ten most influential proteins largely overlapped with those identified in the primary analysis (8/10), with ESR1 being the second ranking molecule (Suppementary Table 5).

Data were available for 238 SNPs linked to 275 genes which also allowed us to perform a subgroup analysis dedicated to estrogen receptor negative breast cancer (only four SNPs were available for estrogen receptor positive cases). Pathway and network analysis yielded results very similar to those obtained in the primary analysis where all breast cancer cases (both receptor positive and negative) were included (data not shown), likely because of the high degree of overlapping between the SNPs (and consequently of genes) of the two series.

\section{SNPS shared with other tumors}

Finally, we assessed whether some breast cancer risk associated SNPs are shared with other malignancies, a phenomenon known as pleiotropy (65). Querying the GWAS Catalog, we found 37 breast cancer risk SNPs shared with other eight tumor types (details are reported in Table 3): ovarian carcinoma $(n=7)$, prostate carcinoma $(n=4)$, lung carcinoma $(n=2)$, thyroid carcinoma $(n=1)$, esophageal carcinoma $(n=1)$, renal cell carcinoma $(n=1)$, cutaneous melanoma $(n=1)$, glioma/glioblastoma $(n=1)$ and a tumor miscellany mainly including ovarian, prostate and lung carcinoma $(n=28)$. In two cases, the breast cancer susceptibility locus was shared with other three tumor types: one SNP (rs13016963) was located in chromosome 2q33.1 (sharing tumors: prostate and esophageal carcinomas, and cutaneous melanoma), the other SNP (rs10069690) in chromosome 5p15.33 (sharing tumors: ovarian and thyroid carcinomas, and glioma/glioblastoma).

These shared SNPs were associated with 34 genes: when we input these BCPGs into a pathway analysis, enrichment in apoptosis and cancer-related pathways was observed (Supplementary Table 6). Upon network analysis, the connectivity was very low (observed edges: 2; expected edges: 1; PPI enrichment P-value: 
bioRxiv preprint doi: https://doi.org/10.1101/279984; this version posted March 12, 2018. The copyright holder for this preprint (which was not certified by peer review) is the author/funder, who has granted bioRxiv a license to display the preprint in perpetuity. It is made available under aCC-BY-NC-ND 4.0 International license.

0.386). Adding 10 first-shell interactors showed the enrichment in cancer-related pathways as well as ribosome machinery and degenerative disease pathways (Supplementary Table 6).

Table 3: Breast cancer risk associated single nucleotide polymorphisms (SNPs) shared with other malignancies

\begin{tabular}{|c|c|c|c|}
\hline Cancer & Chromosome & SNP & Genes \\
\hline Glioma/Glioblastoma & $5 \mathrm{p} 15.33$ & rs 10069690 & TERT \\
\hline Ovarian carcinoma & $5 p 15.33$ & rs10069690 & TERT \\
\hline Thyroid carcinoma & $5 p 15.33$ & rs 10069690 & TERT \\
\hline Miscellany & $9 p 21.3$ & rs1011970 & CDKN2B \\
\hline Prostate carcinoma & $9 p 21.3$ & rs1011970 & CDKN2B \\
\hline Miscellany & $19 \mathrm{p} 13.11$ & rs10419397 & ABHD8;ANKLE1;BABAM1 \\
\hline Ovarian carcinoma & 19p13.11 & rs10419397 & ABHD8;ANKLE1;BABAM1 \\
\hline Miscellany & $14 q 24.1$ & rs10483813 & RAD51B \\
\hline Miscellany & $10 q 26.13$ & rs1078806 & FGFR2 \\
\hline Miscellany & $10 q 26.13$ & rs11200014 & FGFR2 \\
\hline Lung carcinoma & $13 q 13.1$ & rs11571833 & BRCA2 \\
\hline Miscellany & $14 q 24.1$ & rs118446632 & RAD51B \\
\hline Miscellany & $20 q 11.22$ & rs 11907546 & CHMP4B \\
\hline Miscellany & $10 q 26.13$ & rs1219648 & FGFR2 \\
\hline Esophageal carcinoma & $2 q 33.1$ & rs13016963 & CASP8;ALS2CR12 \\
\hline Melanoma (cutaneous) & $2 q 33.1$ & rs13016963 & CASP8;ALS2CR12 \\
\hline Prostate carcinoma & $2 q 33.1$ & rs13016963 & CASP8;ALS2CR12 \\
\hline Miscellany & $3 p 24.1$ & rs1352941 & NEK10;SLC4A7 \\
\hline Miscellany & $19 \mathrm{p} 13$ & rs1469713 & MAU2;SUGP1;NDUFA13;GATAD2A;CILP2;TM6SF2 \\
\hline Prostate carcinoma & $5 p 15.33$ & rs2242652 & TERT \\
\hline Ovarian carcinoma & 19p13.11 & rs2363956 & ABHD8;ANKLE1;MRPL34;OCEL1 \\
\hline Miscellany & $3 p 24.1$ & rs2590265 & NEK10 \\
\hline Miscellany & $10 q 26.13$ & rs2912780 & FGFR2 \\
\hline Miscellany & $10 q 26.13$ & rs2981575 & FGFR2 \\
\hline Miscellany & $10 \mathrm{q} 26.13$ & rs2981582 & FGFR2 \\
\hline Miscellany & $10 \mathrm{q} 26.13$ & rs3135718 & FGFR2 \\
\hline Prostate carcinoma & $1 q 32.1$ & rs4245739 & MDM4;PIK3C2B \\
\hline Miscellany & $19 \mathrm{p} 13.11$ & rs4808075 & ANO8;ABHD8;ANKLE1;BABAM1;OCEL1 \\
\hline Ovarian carcinoma & $19 \mathrm{p} 13.11$ & rs4808075 & ANO8;ABHD8;ANKLE1;BABAM1;OCEL1 \\
\hline Miscellany & $3 p 24.1$ & rs481519 & NEK10 \\
\hline Miscellany & $3 p 24.1$ & rs571978 & NEK10 \\
\hline Miscellany & $3 p 24.1$ & rs580057 & NEK10 \\
\hline Miscellany & $5 q 11.2$ & rs59957907 & C5orf67;MAP3K1 \\
\hline
\end{tabular}


bioRxiv preprint doi: https://doi org/101101/279984; this version posted March 12, 2018. The copyright holder for this preprint (which was not certified by peer review) is the author/funder, who has granted bioRxiv a license to display the preprint in perpetuity. It is made available under aCC-BY-NC-ND 4.0 International license.

\begin{tabular}{|c|c|c|c|}
\hline Miscellany & 19p13.11 & rs61494113 & ABHD8;ANKLE1;OCEL1 \\
\hline Ovarian carcinoma & 19p13.11 & rs61494113 & ABHD8;ANKLE1;OCEL1 \\
\hline Miscellany & $14 q 24.1$ & rs61986943 & RAD51B \\
\hline Ovarian carcinoma & $9 p 34$ & rs635634 & SURF6;ABO \\
\hline Miscellany & $5 q 11.2$ & rs6450401 & MAP3K1;SETD9 \\
\hline Miscellany & $5 q 11.2$ & rs6890270 & MIER3;SETD9 \\
\hline Miscellany & $14 q 24.1$ & rs71423318 & RAD51B \\
\hline Renal cell carcinoma & $11 \mathrm{q} 22.3$ & rs74911261 & KDELC2 \\
\hline Miscellany & $5 q 11.2$ & rs7709971 & C5orf67;MAP3K1 \\
\hline Miscellany & $5 q 11.2$ & rs7714232 & C5orf67;MAP3K1 \\
\hline Lung carcinoma & $5 p 15.33$ & rs7726159 & TERT \\
\hline Miscellany & $5 \mathrm{p} 15.33$ & rs7726159 & TERT \\
\hline Ovarian carcinoma & 19p13.11 & rs8170 & PLVAP;NR2F6;BABAM1;MRPL34;USHBP1;ABHD8;ANKLE1 \\
\hline
\end{tabular}




\section{Discussion}

We reported on the first knowledge-base dedicated on GWAS-based evidence linking common germline variants to the risk of breast cancer. The information on breast cancer risk associated SNPs forms a knowledge-base which will be made publicly available at our cancer dedicated website (www.mmmp.org (66)) and will be annually updated.

Following the principles of integrative analysis of genomic data, we combined genome-wide information from different sources (e.g., high-throughput genotyping experiments, eQTL analysis, LD analysis, and so on) to make the most of the available evidence $(8,9)$. This is of particular relevance because most SNPs do not have a direct functional effect, indeed a large proportion of associated SNPs are not in the coding regions of genes, and thus additional information is needed to link them to a gene. Then, we used these data to make tentative inferences on the pathways (and most influential molecules within them) whose variation can affect the risk of developing breast cancer.

Data from almost 400,000 women affected with breast cancer showed that 281 SNPs are significantly associated with the risk of this disease, which reduced to 233 risk loci when linkage disequilibrium was taken into account. These findings add new information to the already existing recent literature reviews on this subject, which report up to 172 common variants linked to breast cancer susceptibility $(3,4,6,67-71)$. These SNPs are estimated to account $15-20 \%$ of the genetic component of disease risk $(3,72)$, which clearly implies that much more work is needed to fully elucidate the molecular basis of breast cancer predisposition. It has been argued that future GWAS will not lead to the discovery of many more risk variants (3). This appears especially true in terms of rare variants (that is, variants with a MAF $<1 \%)(3,72)$, as GWAS studies are designed to identify only common polymorphisms (MAF $>1 \%$ ) through a tagging strategy (tested tag-SNPs are in high linkage disequilibrium with non-tested SNPs). Moving forward, massively parallel sequencing technology (a.k.a. next generation sequencing [NGS], which can directly interrogate every genomic position) could provide investigators with the right tool to overcome the challenging hurdle of interrogating rarer variants which may affect risk, thus adding essential information to this field of investigation (73). 
bioRxiv preprint doi: https://doi.org/10.1101/279984; this version posted March 12, 2018. The copyright holder for this preprint (which was not certified by peer review) is the author/funder, who has granted bioRxiv a license to display the preprint in perpetuity. It is made available under aCC-BY-NC-ND 4.0 International license.

The data collected in our knowledge-base can be used to build polygenic predictive models and thus help optimize breast cancer secondary prevention programs (i.e. early detection by mammographic screening) by selecting women at higher risk (74-77). So far, such models have yielded generally unsatisfactory results, as their accuracy remains too low to be clinically implemented. This could be due to the fact that the complex genetic architecture of sporadic breast cancer predisposition remains still to be fully elucidated, as well as to the lack of information on gene-environment interactions $(78,79)$. Nevertheless, the systematic collection of variants associated with breast cancer risk, along with information on their functional effect (as proposed in our knowledge-base) is the first step to build more effective predictive tools.

We utilized the collected information to generate tentative mechanistic hypotheses on the pathways whose perturbation (as determined by germline variation of the corresponding genes) affect breast cancer susceptibility. Some studies have already investigated the role of the variation of a single pathway across the results of multiple GWAS or the variation of multiple pathways within a single GWAS in the determinism of breast carcinogenesis $(80,81)$. However, to the best of our knowledge, this is the first time that the comprehensive collection of variants linked to breast cancer risk by means of all available GWAS (and their meta-analyses) has been employed to systematically explore the cell pathways potentially involved in breast cancer development. Our gene set enrichment analysis led to the identification of multiple pathways well known to be involved in cancer development in general (such as apoptosis, cell cycle, and signal transduction) and breast cancer in particular (such as steroid hormone pathways). As regards the latter, the estrogen receptor pathway was confirmed to play a pivotal role in the carcinogenesis of a hormone dependent neoplasm such as breast carcinoma (82), within this frame, the gene encoding the estrogen receptor alpha (ESR1) was a key influencer in the generated networks of BCPGs (see Figure 1 and Supplementary Table 5). This finding might be of relevance with regard to breast cancer chemoprevention, which aims to reduce disease incidence by the administration of anti-estrogen drugs such as selective estrogen receptor modifiers (e.g., tamoxifen) (83). The selection of women who could most benefit from these risk reducing medications might be improved by genetic testing based on polymorphisms that affect breast cancer risk (84). 
bioRxiv preprint doi: https://doi.org/10.1101/279984 - this version posted March 12, 2018. The copyright holder for this preprint (which was not certified by peer review) is the author/funder, who has granted bioRxiv a license to display the preprint in perpetuity. It is made available under aCC-BY-NC-ND 4.0 International license.

Another interesting piece of information yielded from data analysis is the high degree of overlap between network-guided gene set enrichment primary analysis and the pathway analysis performed with the targets of BCSGs acting as transcription factors (see Table 1 and Table 2). This finding supports the hypothesis that most of the biological effect of the SNPs linked to breast cancer risk might actually be mediated by regulons governed by the transcription factors associated with those SNPs. Notably, our data confirm the results of a recent publication where investigators have identified a breast cancer risk regulatory network comprising some of the transcription factors we identified as BCPGs (85).

Besides well known cancer-related pathways (such as apoptosis, signal transduction and so on), our gene set enrichment analysis showed that germline variation of other pathways might be of particular relevance for breast cancer susceptibility, such as those involved in anti-viral immunity, degenerative diseases as well as peroxisome and ribosome activity (see Table 1 and Table 2). Actually, peroxisomes are known to be linked to carcinogenesis through their production of reactive oxygen species (86), which in turn can initiate tumor development by causing DNA damage. Of special interest is also the case of genes encoding ribosome proteins, which were repeatedly enriched in our pathway and network analyses of the whole series, as well as in the analysis of pleiotropic SNPs. Indeed, it has recently been suggested that ribosome derangement may play a significant role in both development and progression of different tumor types $(87,88)$, including breast cancer (89).

In conclusion, we present the first knowledge-base dedicated to sporadic breast cancer predisposition variants. This wealth of information can inform future studies aimed to dissect the molecular epidemiology and the molecular basis of this disease. 


\section{References}

1) DeSantis CE, Bray F, Ferlay J, et al. International Variation in Female Breast Cancer Incidence and Mortality Rates. Cancer Epidemiol Biomarkers Prev 2015;24(10):1495-506.

2) Senft D, Leiserson MDM, Ruppin E, et al. Precision Oncology: The Road Ahead. Trends Mol Med 2017;23(10):874-898

3) Skol AD, Sasaki MM, Onel K. The genetics of breast cancer risk in the post-genome era: thoughts on study design to move past BRCA and towards clinical relevance. Breast Cancer Res 2016;18(1):99.

4) Ghoussaini M, Pharoah PDP, Easton DF. Inherited genetic susceptibility to breast cancer: the beginning of the end or the end of the beginning? Am J Pathol 2013;183(4):1038-1051.

5) Zhang B, Beeghly-Fadiel A, Long J, et al. Genetic variants associated with breast-cancer risk: comprehensive research synopsis, meta-analysis, and epidemiological evidence. Lancet Oncol 2011;12(5):477-88.

6) Fachal L, Dunning AM. From candidate gene studies to GWAS and post-GWAS analyses in breast cancer. Curr Opin Genet Dev 2015;30:32-41.

7) MacArthur J, Bowler E, Cerezo M, et al. The new NHGRI-EBI Catalog of published genome-wide association studies (GWAS Catalog). Nucleic Acids Res 2017;45(D1):D896-D901.

8) Kristensen VN, Lingjaerde OC, Russnes HG, et al. Principles and methods of integrative genomic analyses in cancer. Nat Rev Cancer 2014;14:299-313.

9) Thingholm LB, Andersen L, Makalic E, et al. Strategies for Integrated Analysis of Genetic, Epigenetic, and Gene Expression Variation in Cancer: Addressing the Challenges. Front Genet 2016;7:2.

10) Mooney MA, Nigg JT, McWeeney SK, et al. Functional and genomic context in pathway analysis of GWAS data. Trends Genet 2014;30(9):390-400.

11) Creixell P, Reimand J, Haider S, et al. Pathway and network analysis of cancer genomes. Nat Methods 2015;12:615-621.

12) The GTEx Consortium. Genetic effects on gene expression across human tissues. Nature 2017;550:204-213. 
13) Ernst J, Kheradpour P, Mikkelsen TS, et al. Mapping and analysis of chromatin state dynamics in nine human cell types. Nature 2011;473(7345):43-9.

14) Bernstein BE, Stamatoyannopoulos JA, Costello JF, et al. The NIH Roadmap Epigenomics Mapping Consortium. Nat Biotechnol 2010;28(10):1045-8.

15) Boyle AP, Hong EL, Hariharan M, et al. Annotation of functional variation in personal genomes using RegulomeDB. Genome Res 2012;22(9):1790-7.

16) Ward LD, Kellis M. HaploReg: a resource for exploring chromatin states, conservation, and regulatory motif alterations within sets of genetically linked variants. Nucleic Acids Res 2012;40(Database issue):D930-4.

17) Machiela MJ, Chanock SJ. LDlink: a web-based application for exploring population-specific haplotype structure and linking correlated alleles of possible functional variants. Bioinformatics 2015;31(21):3555-7.

18) Kuleshov MV, Jones MR, Rouillard AD, et al. Enrichr: a comprehensive gene set enrichment analysis web server 2016 update. Nucleic Acids Res 2016;44(W1):W90-7.

19) Rivals I, Personnaz L, Taing L, et al. Enrichment or depletion of a GO category within a class of genes: which test? Bioinformatics 2007;23(4):401-7.

20) Storey JD, Tibshirani R. Statistical significance for genomewide studies. Proc Natl Acad Sci U S A 2003;100(16):9440-5.

21) Szklarczyk D, Morris JH, Cook H, et al. The STRING database in 2017: quality-controlled proteinprotein association networks, made broadly accessible. Nucleic Acids Res 2017;45:D362-68.

22) He X, Zhang J. Why do hubs tend to be essential in protein networks? PLoS Genet 2006;2(6):e88.

23) Bean DM, Heimbach J, Ficorella L, et al. EsyN: network building, sharing and publishing. PLoS One 2014;9(9):e106035.

24) Morone F, Makse HA. Influence maximization in complex networks through optimal percolation. Nature 2015;524(7563):65-8. 
25) UniProt Consortium. UniProt: a hub for protein information. Nucleic Acids Res 2015;43(Database issue):D204-12.

26) Han $\mathrm{H}$, Cho JW, Lee $\mathrm{S}$, et al. TRRUST v2: an expanded reference database of human and mouse transcriptional regulatory interactions. Nucleic Acids Res 2018;46(D1):D380-D386.

27) Easton DF, Pooley KA, Dunning AM, et al. Genome-wide association study identifies novel breast cancer susceptibility loci. Nature 2007;447(7148):1087-93.

28) Hunter DJ, Kraft $P$, Jacobs KB, et al. A genome-wide association study identifies alleles in FGFR2 associated with risk of sporadic postmenopausal breast cancer. Nat Genet 2007;39(7):870-4.

29) Stacey SN, Manolescu A, Sulem P, et al. Common variants on chromosomes $2 q 35$ and $16 q 12$ confer susceptibility to estrogen receptor-positive breast cancer. Nat Genet 2007;39(7):865-9.

30) Gold B, Kirchhoff T, Stefanov S, et al. Genome-wide association study provides evidence for a breast cancer risk locus at 6q22.33. Proc Natl Acad Sci U S A 2008;105(11):4340-5.

31) Zheng W, Long J, Gao YT, et al. Genome-wide association study identifies a new breast cancer susceptibility locus at 6q25.1. Nat Genet 2009;41(3):324-8.

32) Thomas $G$, Jacobs KB, Kraft $P$, et al. A multistage genome-wide association study in breast cancer identifies two new risk alleles at 1p11.2 and 14q24.1 (RAD51L1). Nat Genet 2009;41(5):579-84.

33) Turnbull C, Ahmed S, Morrison J, et al. Genome-wide association study identifies five new breast cancer susceptibility loci. Nat Genet 2010;42(6):504-7.

34) Long J, Cai Q, Shu XO, et al. Identification of a functional genetic variant at $16 q 12.1$ for breast cancer risk: results from the Asia Breast Cancer Consortium. PLoS Genet 2010;6(6):e1001002.

35) Antoniou AC, Wang X, Fredericksen ZS, et al. A locus on 19p13 modifies risk of breast cancer in BRCA1 mutation carriers and is associated with hormone receptor-negative breast cancer in the general population. Nat Genet 2010;42(10):885-92.

36) Li J, Humphreys K, Heikkinen T, et al. A combined analysis of genome-wide association studies in breast cancer. Breast Cancer Res Treat 2011;126(3):717-27. 
37) Gaudet MM, Kirchhoff T, Green T, et al. Common genetic variants and modification of penetrance of BRCA2-associated breast cancer. PLoS Genet 2010;6(10):e1001183.

38) Fletcher $\mathrm{O}$, Johnson $\mathrm{N}$, Orr $\mathrm{N}$, et al. Novel breast cancer susceptibility locus at 9q31.2: results of a genome-wide association study. J Natl Cancer Inst 2011;103(5):425-35.

39) Cai Q, Long J, Lu W, et al. Genome-wide association study identifies breast cancer risk variant at 10q21.2: results from the Asia Breast Cancer Consortium. Hum Mol Genet 2011;20(24):4991-9.

40) Haiman CA, Chen GK, Vachon CM, et al. A common variant at the TERT-CLPTM1L locus is associated with estrogen receptor-negative breast cancer. Nat Genet 2011;43(12):1210-4.

41) Long J, Cai $Q$, Sung $H$, et al. Genome-wide association study in east Asians identifies novel susceptibility loci for breast cancer. PLoS Genet 2012;8(2):e1002532.

42) Kim HC, Lee JY, Sung $\mathrm{H}$, et al. A genome-wide association study identifies a breast cancer risk variant in ERBB4 at 2q34: results from the Seoul Breast Cancer Study. Breast Cancer Res 2012;14(2):R56.

43) Elgazzar S, Zembutsu $H$, Takahashi $A$, et al. A genome-wide association study identifies a genetic variant in the SIAH2 locus associated with hormonal receptor-positive breast cancer in Japanese. J Hum Genet 2012;57(12):766-71.

44) Siddiq A, Couch FJ, Chen GK, et al. A meta-analysis of genome-wide association studies of breast cancer identifies two novel susceptibility loci at 6q14 and 20q11. Hum Mol Genet 2012;21(24):537384.

45) Orr N, Lemnrau A, Cooke R, et al. Genome-wide association study identifies a common variant in RAD51B associated with male breast cancer risk. Nat Genet 2012;44(11):1182-4.

46) Rinella ES, Shao Y, Yackowski L, et al. Genetic variants associated with breast cancer risk for Ashkenazi Jewish women with strong family histories but no identifiable BRCA1/2 mutation. Hum Genet 2013;132(5):523-36.

47) Michailidou K, Hall P, Gonzalez-Neira A, et al. Large-scale genotyping identifies 41 new loci associated with breast cancer risk. Nat Genet 2013;45(4):353-61. 
48) Garcia-Closas M, Couch FJ, Lindstrom S, et al. Genome-wide association studies identify four ER negative-specific breast cancer risk loci. Nat Genet 2013;45(4):392-8.

49) Couch FJ, Wang $X$, McGuffog $L$, et al. Genome-wide association study in BRCA1 mutation carriers identifies novel loci associated with breast and ovarian cancer risk. PLoS Genet 2013;9(3):e1003212.

50) Low SK, Takahashi A, Ashikawa K, et al. Genome-wide association study of breast cancer in the Japanese population. PLoS One 2013;8(10):e76463.

51) Purrington KS, Slager $S$, Eccles $D$, et al. Genome-wide association study identifies 25 known breast cancer susceptibility loci as risk factors for triple-negative breast cancer. Carcinogenesis 2014;35(5):1012-9.

52) Ahsan H, Halpern J, Kibriya MG, et al. A genome-wide association study of early-onset breast cancer identifies PFKM as a novel breast cancer gene and supports a common genetic spectrum for breast cancer at any age. Cancer Epidemiol Biomarkers Prev 2014;23(4):658-69.

53) Sawyer E, Roylance R, Petridis C, et al. Genetic predisposition to in situ and invasive lobular carcinoma of the breast. PLoS Genet 2014;10(4):e1004285.

54) Cai Q, Zhang B, Sung H, et al. Genome-wide association analysis in East Asians identifies breast cancer susceptibility loci at 1q32.1, 5q14.3 and 15q26.1. Nat Genet 2014;46(8):886-90.

55) Fejerman L, Ahmadiyeh N, Hu D, et al. Genome-wide association study of breast cancer in Latinas identifies novel protective variants on 6q25. Nat Commun 2014;5:5260.

56) Michailidou K, Beesley J, Lindstrom S, et al. Genome-wide association analysis of more than 120,000 individuals identifies 15 new susceptibility loci for breast cancer. Nat Genet 2015;47(4):373-80.

57) Palomba G, Loi A, Porcu E, et al. Genome-wide association study of susceptibility loci for breast cancer in Sardinian population. BMC Cancer 2015;15:383.

58) Couch FJ, Kuchenbaecker KB, Michailidou K, et al. Identification of four novel susceptibility loci for oestrogen receptor negative breast cancer. Nat Commun 2016;7:11375. 
59) Fehringer G, Kraft P, Pharoah PD, et al. Cross-Cancer Genome-Wide Analysis of Lung, Ovary, Breast, Prostate, and Colorectal Cancer Reveals Novel Pleiotropic Associations. Cancer Res 2016;76(17):5103-14.

60) Han MR, Long J, Choi JY, et al. Genome-wide association study in East Asians identifies two novel breast cancer susceptibility loci. Hum Mol Genet 2016;25(15):3361-3371.

61) Kar SP, Beesley J, Amin Al Olama A, et al. Genome-Wide Meta-Analyses of Breast, Ovarian, and Prostate Cancer Association Studies Identify Multiple New Susceptibility Loci Shared by at Least Two Cancer Types. Cancer Discov 2016;6(9):1052-67.

62) Huo D, Feng Y, Haddad S, et al. Genome-wide association studies in women of African ancestry identified 3q26.21 as a novel susceptibility locus for oestrogen receptor negative breast cancer. Hum Mol Genet 2016;25(21):4835-4846.

63) Michailidou K, Lindström S, Dennis J, et al. Association analysis identifies 65 new breast cancer risk loci. Nature 2017;551(7678):92-94.

64) Milne RL, Kuchenbaecker KB, Michailidou K, et al. Identification of ten variants associated with risk of estrogen-receptor-negative breast cancer. Nat Genet 2017;49(12):1767-1778.

65) Wu YH, Graff RE, Passarelli MN, et al. Identification of Pleiotropic Cancer Susceptibility Variants from Genome-Wide Association Studies Reveals Functional Characteristics. Cancer Epidemiol Biomarkers Prev 2018;27(1):75-85.

66) Mocellin S, Rossi CR. The melanoma molecular map project. Melanoma Res 2008;18(3):163-5.

67) Peng S, Lü B, Ruan W, et al. Genetic polymorphisms and breast cancer risk: evidence from metaanalyses, pooled analyses, and genome-wide association studies. Breast Cancer Res Treat 2011;127(2):309-24.

68) Maxwell KN, Nathanson KL. Common breast cancer risk variants in the post-COGS era: a comprehensive review. Breast Cancer Res 2013;15(6):212.

69) Sapkota Y. Germline DNA variations in breast cancer predisposition and prognosis: a systematic review of the literature. Cytogenet Genome Res 2014;144(2):77-91. 
70) Walsh MF, Nathanson KL, Couch FJ, et al. Genomic Biomarkers for Breast Cancer Risk. Adv Exp Med Biol 2016;882:1-32

71) Lilyquist J, Ruddy KJ, Vachon CM, et al. Common Genetic Variation and Breast Cancer Risk - Past, present, and future. Cancer Epidemiol Biomarkers Prev 2018; [Epub ahead of print]

72) Zuk O, Schaffner SF, Samocha K, et al. Searching for missing heritability: designing rare variant association studies. Proc Natl Acad Sci U S A 2014;111(4):E455-64.

73) Chandler MR, Bilgili EP, Merner ND. A Review of Whole-Exome Sequencing Efforts Toward Hereditary Breast Cancer Susceptibility Gene Discovery. Hum Mutat 2016;37(9):835-46.

74) Evans DG, Brentnall A, Byers $\mathrm{H}$, et al. The impact of a panel of 18 SNPs on breast cancer risk in women attending a UK familial screening clinic: a case-control study. J Med Genet 2017;54(2):111-113.

75) Shieh Y, Hu D, Ma L, et al. Breast cancer risk prediction using a clinical risk model and polygenic risk score. Breast Cancer Res Treat 2016;159(3):513-25.

76) Mavaddat N, Pharoah PD, Michailidou K, et al. Prediction of breast cancer risk based on profiling with common genetic variants. J Natl Cancer Inst 2015;107(5).

77) Cuzick J, Brentnall AR, Segal C, et al. Impact of a Panel of 88 Single Nucleotide Polymorphisms on the Risk of Breast Cancer in High-Risk Women: Results From Two Randomized Tamoxifen Prevention Trials. J Clin Oncol 2017;35(7):743-750.

78) Rudolph A, Song $M$, Brook MN, et al. Joint associations of a polygenic risk score and environmental risk factors for breast cancer in the Breast Cancer Association Consortium. Int J Epidemiol 2018; [Epub ahead of print]

79) Ito H, Matsuo K. Molecular epidemiology, and possible real-world applications in breast cancer. Breast Cancer 2016;23(1):33-8.

80) Lee YH, Kim JH, Song GG. Genome-wide pathway analysis of breast cancer. Tumour Biol 2014;35(8):7699-705. 
81) Scarbrough PM, Weber RP, Iversen ES, et al. A Cross-Cancer Genetic Association Analysis of the DNA Repair and DNA Damage Signaling Pathways for Lung, Ovary, Prostate, Breast, and Colorectal Cancer. Cancer Epidemiol Biomarkers Prev 2016;25(1):193-200.

82) Li T, Zhao J, Yang J, et al. A Meta-Analysis of the Association between ESR1 Genetic Variants and the Risk of Breast Cancer. PLoS One 2016;11(4):e0153314.

83) Mocellin S, Pilati P, Briarava M, et al. Breast Cancer Chemoprevention: A Network Meta-Analysis of Randomized Controlled Trials. J Natl Cancer Inst 2015;108(2). pii: djv318.

84) Ziv E, Tice JA, Sprague B, et al. Using Breast Cancer Risk Associated Polymorphisms to Identify Women for Breast Cancer Chemoprevention. PLoS One 2017;12(1):e0168601.

85) Castro MA, de Santiago I, Campbell TM, et al. Regulators of genetic risk of breast cancer identified by integrative network analysis. Nat Genet 2016;48(1):12-21.

86) Gào X, Schöttker B. Reduction-oxidation pathways involved in cancer development: a systematic review of literature reviews. Oncotarget 2017;8(31):51888-51906.

87) Pelletier J, Thomas G, Volarević S. Ribosome biogenesis in cancer: new players and therapeutic avenues. Nat Rev Cancer 2018;18(1):51-63.

88) Bustelo XR, Dosil M. Ribosome biogenesis and cancer: basic and translational challenges. Curr Opin Genet Dev 2017;48:22-29.

89) Belin S, Beghin A, Solano-Gonzàlez E, et al. Dysregulation of ribosome biogenesis and translational capacity is associated with tumor progression of human breast cancer cells. PLoS One 2009;4(9):e7147. 